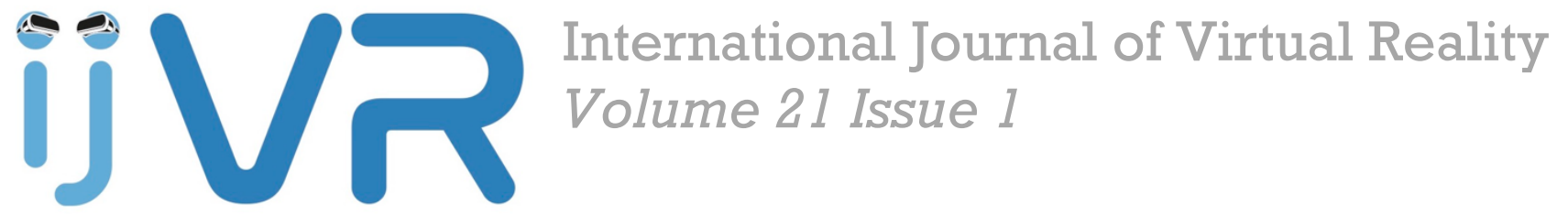

Cherni, H., Nicolas, S., \& Métayer, N. (2021).

Using virtual reality treadmill as a locomotion technique in a navigation task: Impact on user experience - case of the KatWalk.

International Journal of Virtual Reality, 21(1), 1-14.

https://doi.org/ 10.20870/IJVR.2021.21.1.3046

CC BY 4.0 license

Published: 5/2021

ISSN: 2727-9979 


\title{
Using virtual reality treadmill as a locomotion technique in a navigation task: Impact on user experience - case of the KatWalk
}

\author{
Heni Cherni ${ }^{1}$, Nicolas Souliman ${ }^{1}$, Natacha Metayer ${ }^{1}$ \\ ${ }^{1}$ Institut VEDECOM, 23 bis allée des marronniers, 78000 Versailles
}

Corresponding author: Heni Cherni, heni.cherni@vedecom.fr

Keywords: Virtual reality - Locomotion techniques - Navigation - Omnidirectional treadmill

\begin{abstract}
This paper reports empirical results from a study on the impact of the KatWalk virtual reality omnidirectional treadmill on the user experience. The omnidirectional treadmill is a mechanical device, that allows the user to perform locomotive motion in any direction, allowing for 360 degrees of horizontal movement. This locomotion method appeared as a potential solution of the locomotion problem in virtual reality after the emergence and the democratization of the new generation of head-mounted display systems such as HTC Vive and Oculus Rit. However, little empirical work has been done to test the efficiency of the tool as a locomotion technique in virtual environments. Twenty-four subjects (13 females, mean age $=30.38$, SD $=6.32$ ) participated in the experiment. Results showed that the tool is suitable for traveling in virtual environments seen through head-mounted display systems, whether they are composed of plan or bumpy ground, with or without obstacles.
\end{abstract}

\section{Introduction}

Virtual reality allows the user to extract him/herself from a real environment into a virtual environment where he/she can observe, interact, dialogue and even learn new skills. This flexibility has opened new horizons in several areas such as robotics, urban planning, industry, art and education, etc. For example, an aircraft pilot can practice to land safely in in different inclement weather conditions, without endangering the crew nor the passengers. A surgeon may be trained for a specific intervention, before actually performing it. In addition, there are situations that are difficult to reproduce in real world, such as a train derailment or a surgical complication. Users can train and repeat complex situations at will in a virtual and controllable environment (Cherni, 2012; Ohshima et al., 2016).

Over the last ten years, technological progress has significantly improved user experience (UX) in virtual reality (VR) (Grechkin et al., 2014). With the emergence and the democratisation of affordable head-mounted display systems like HTC Vive and Oculus Rift, common locomotion devices such as joysticks have been shown to cause disorientation in immersive virtual environments (VE) (Davis et al., 2014). Virtual reality locomotion enables the user to move in an infinite-scale virtual-world by doing real walking movements, while staying physically confined in a room-scale real-world environment (Hale \& Stanney, 2014). Locomotion is an essential form of human machine interaction. In virtual reality, it has a direct effect on many aspects of user experience such as enjoyment, frustration, tiredness, motion sickness and presence. When the virtual environment is the same size as the physical environment of the user and the system allows to move around freely, the user can walk naturally to 
control the virtual-world locomotion. However, when the virtual environment is larger than the physical environment, an alternative locomotion method must be used. Despite the fact that locomotion is not the primary objective of virtual reality applications, it is considered to be one of the most important components of interaction in virtual reality experiences as it is needed for virtual environment exploration (Bowman et al., 2004). The choice of the locomotion technique (LT) for head mounted displays (HMD) is still untapped in terms of understanding, development, use and experimentation. Thus, finding an efficient LT that does not cause motion sickness and avoid fatigue represents a real challenge that has recently become the object of numerous research studies (Bowman et al., 2004; Kitson et al., 2017).

\section{State of the art}

Several studies focused on finding an intuitive and efficient LT. At the most general level, a distinction was identified between two main methods. The first approach uses 3D user controllers to travel in the VE. The second approach, commonly called user body centered method, consists in using the motion capture of different parts of the user's body to determine whether or not the user is walking (Cherni et al., 2020). This technique is also called "leaning based method". In this context, the most common LT is the real walk (Nabiyouni et al., 2015; Tunnell Wilson et al., 2016). The Walking-In-Place method is also one of most used leaning based methods. To travel in the VE, the user engages his/her body in a as much as possible realistic walking motion without actually moving forward (Berger \& Wolf, 2018; Bozgeyikli et al., 2016a; Nilsson et al., 2013). Another leaning based method is the redirected walking (Bozgeyikli et al., 2016a; Bruder et al., 2015). This LT, like the Walking-In-Place method, enables the user to explore a virtual world that is considerably larger than the tracked workspace addressing the limitation of the workspace addressing the limitation of the size of the virtual environment (Zank \& Kunz, 2015). It works by interactively rotating the virtual scene around the user, so that the user is made to continually walk towards the farthest "wall" of the tracker area. Therefore, users are guided unnoticeably on a physical path that differs from the path the user perceives in the virtual world by manipulating the transformations from real to virtual movements (Bruder et al., 2015). This LT exploits the limitations of human perceptual mechanisms for sensing position, orientation and movement, suggesting that the user does not notice the rotation (Razzaque, 2005). However, used with HMD systems, these methods have several limits. For instance, the real walk requires too much space, which most physical environments do not provide (Slater et al., 1995). Same for the Walking-InPlace, it presents three major weak points. The first one relates to the fact that there are many sensors to be attached to the user's body. The second one is that the immersion level is reduced because the user only imitates walking doing unusual gestures. The third weak point is that this technique may not be suitable for exploring a significantly large virtual environment as it would take a considerable amount of time and physical energy (Lee et al., 2018). In the same manner, the redirected walking reduces the sense of presence of the user too much (Bruder et al., 2013; Steinicke et al., 2010).

Other researchers used external peripherals to travel in the virtual environment, such as classic joysticks and trackpad controllers (Kitson et al., 2015), or point and teleport methods which emerged with the HTC Vive controllers (Jacob Habgood et al., 2018; Linn, 2017). Joysticks and trackpads are easy to use and many studies showed that they outperformed and had a better ranking than locomotion techniques such as many leaning based LT (Bozgeyikli et al., 2016a; Jayaraman et al., 2012; McMahan et al., 2012). However, used with a HMD, joysticks may cause a low level of immersion and a high level of motion sickness (Davis et al., 2014). Another LT that immerged with the new generation of HMD as a solution for the locomotion problem in VE is the point and teleport LT. With this technique, users can travel from one place to another by simply pointing the place they want to go to. The new place users wish to travel to is often called "the target location" (Bozgeyikli et al., 2019). In some applications, target locations are predefined, and the user has to point the target to indicate which one he/she wants to reach. In other applications, instead of predefined targets, the users are free to teleport anywhere on the ground by pointing any target position. In both cases, users have often a visual and sometimes an auditive 
feedback to confirm the selected target location as well as the movement to go there (Cherep et al., 2020). The point and teleport technique is known for not introducing motion sickness since it does not involve any visible translational motion, but it may cause disorientation in the VE (Bozgeyikli et al., 2016b; Bozgeyikli et al., 2019). Besides, when pointing the target position, the teleportation can be actioned either by pushing a button or by pointing the target physically for longer than a certain amount of time. In this last case, the use of the arm is "sacrificed". Then, interacting with the virtual environment using the arm is not possible since each time the user rises his hand, it could be construed, by the virtual system, as a command to move through the virtual environment (Bozgeyikli et al., 2016b). Proposition: In this last case, any arm movement risks might be falsely interpreted by the system as a 'pointing-the-new-target' movement. The user might feel hindered in her/his natural way of moving.

Another LT using external peripherals is the Omnidirectional treadmill (ODT) which can be considered as a seminatural LT, referring to the LT taxonomy proposed by Cherni and colleagues (Cherni et al., 2020). This technique was initially designed for gaming purposes. But some researchers quickly realized the potential of this tool as a LT for VR experiments. However, ODT has been the subject of few empirical studies (Barberis et al., 2019; Warren \& Bowman, 2017) but their results are not explained clearly enough, notably the three fundamental challenges of LT in VR which are motion-sickness, presence and fatigue. In their study, Barberis and colleagues tried to identify software parameters for the Cyberith Virtualizer that ensures comfort and performance for the user offering good movement speed or sensor sensitivity (Barberis et al., 2019). In similar purposes, Lawrence and colleagues made an abbreviated comparative study between the joystick and the Virtuix ODT (Warren \& Bowman, 2017). They found that the joystick outperformed the Virtuix ODT without giving details neither on the task nor on the values of the comparison variables.

The aim of this work is to study the impact of using KatWalk ODT on the UX in a navigation task in VR.

\section{Method}

\section{Research questions}

To investigate the effects of the use of the KatWalk ODT on the UX, three key research questions were identified: Q1: Is the KatWalk ODT a usable LT for walking in a VE?

Q2: Is the KatWalk ODT adapted for traveling in a VE with a bumpy ground?

Q3: Is there a difference between walking on plan ground vs. bumpy ground when using the KatWalk ODT as a LT?

\section{Hypothesis}

In order to answer our research questions, we put in place the following 2 hypotheses:

H1: The KatWalk ODT is suitable to travel in VE with bumpy ground. In other words, we hypothesize no difference will appear between both surfaces (i.e. plane and bumpy) for the navigation task and for the number of collisions.

H2: The KatWalk ODT can be used as a LT in virtual environments. In other words, we do not expect any difference between before and after using the KatWalk for SSQ and Self-Assessment Manikin scores. We suppose that this lack of difference on the variables of physical (evaluated by the SSQ) and emotional feelings (evaluated by the SAM) would support our hypothesis on the validity of using the KatWalk as a locomotion tool in VR.

\section{Participants}

Twenty-four participants (13 females and 11 males, mean age $=30.38, S D=6.32$ ) took part in the experiment. All participants had normal or corrected vision. Participants were volunteers and received no gratification. Table 1 
shows the repartition of participants depending on their practice of VR, ODT and video games and interactive worlds in general. Twelve of them used a VR HMD for the first time, and 17 had no practice of ODT.

Table 1. Effective practice of participants

\begin{tabular}{|c|c|c|c|c|}
\hline \multirow[t]{3}{*}{ Practice } & & 2 to & 4 to & \multirow{3}{*}{$\begin{array}{c}\text { Mor } \\
\text { e }\end{array}$} \\
\hline & $\begin{array}{l}\text { First } \\
\text { time }\end{array}$ & $\begin{array}{c}3 \\
\text { time }\end{array}$ & $\begin{array}{c}5 \\
\text { time }\end{array}$ & \\
\hline & & $\mathrm{s}$ & $\mathrm{S}$ & \\
\hline VR this calendar year & 12 & 6 & 3 & 3 \\
\hline ODT this calendar year & 17 & 3 & 1 & 3 \\
\hline $\begin{array}{l}\text { Interactive worlds per } \\
\text { week }\end{array}$ & 6 & 6 & 7 & 5 \\
\hline
\end{tabular}

\section{Instrumentation}

Virtual environment

We designed an uncluttered VE of 50 x 50 meters ground surface surrounded by an enclosure wall. The ground can be either plane or bumpy depending on the experiment conditions. Observed literature seems to report only on experimentations in VE with plane ground and does not consider irregularities of height. To prepare further experimentations in urban VE, our research team shows an interest on the reliability of LT on irregular ground.

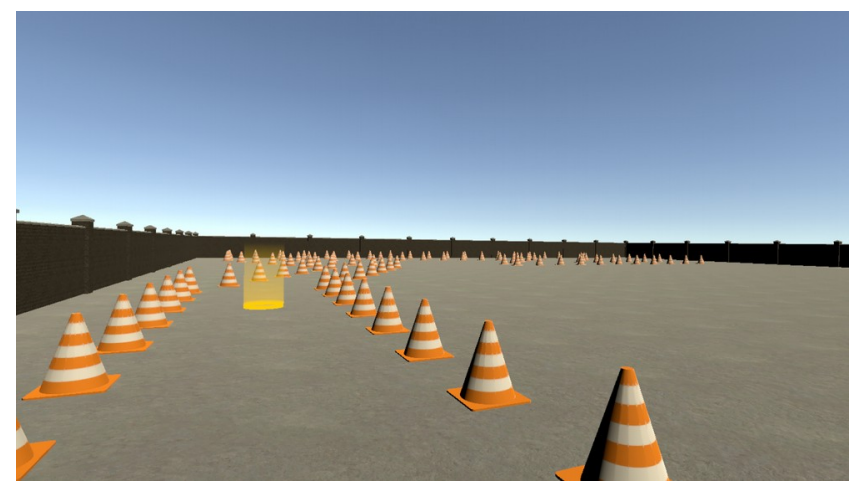

Figure 1. Virtual environment from the participant point of view for the condition with plane surface in presence of obstacles.

Depending on the experimental conditions, obstacles (80 cm high traffic cones) are disposed shaping a 3.4 meters wide path, drawing a curve between the first and the second point (see Figures 1 and 2). The bumpy surface environment has been chosen to simulate the vertical amplitude that participants can encounter in an urban environment such as sidewalks crossing, road degradation or small steps (Figure 3). 


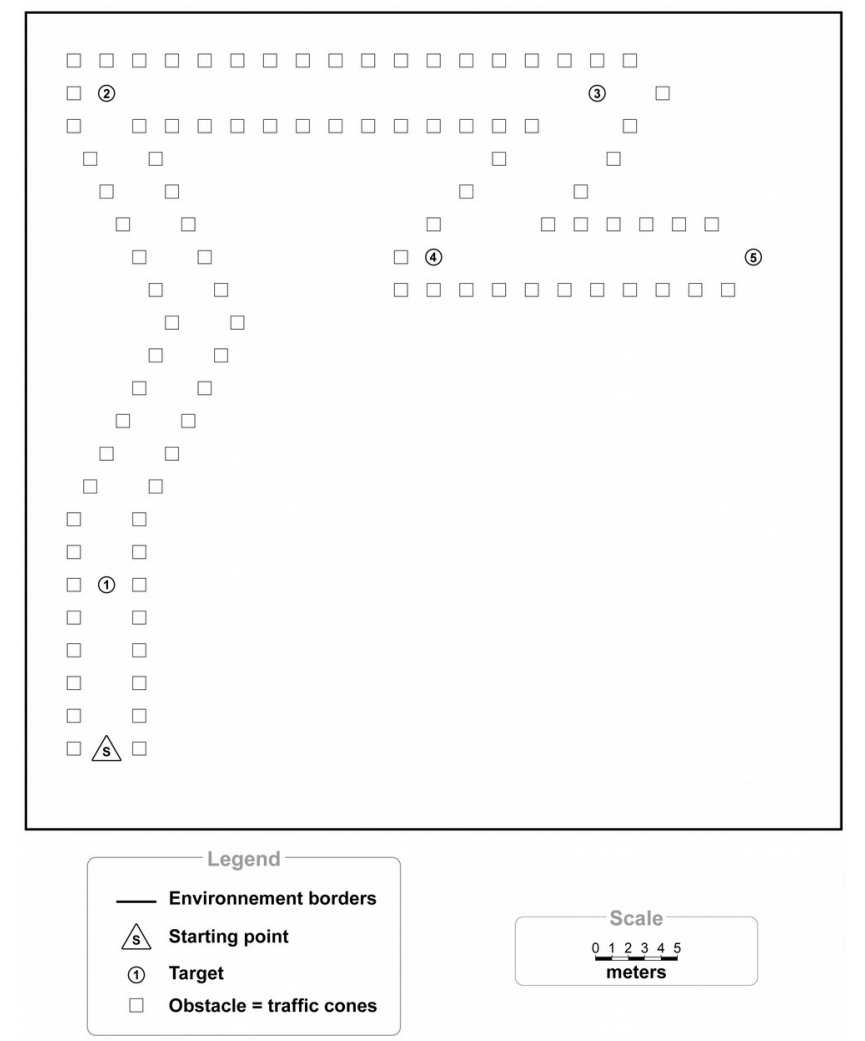

Figure 2. Virtual environment map.

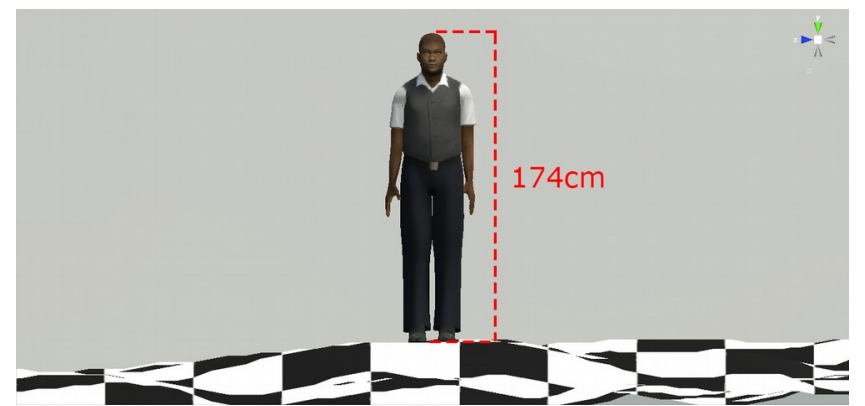

Figure 3. the bumpy surface environment textured with checkerboard to highlight irregularities

\section{Display System}

The VE was displayed on HTC Vive HMD. The HTC Vive is a virtual reality HMD developed by HTC and Valve. The headset uses room scale tracking technology, allowing the user to move in 3D space and to use motiontracked handheld controllers to interact with the environment. It uses two 1200 x 1080 px displays that refresh at 90 frames per second. It is considered to be the first device to offer a full room-scale experience, letting the user to get up, to walk around and explore the virtual space, to inspect objects from every angle and to interact with the surroundings (The verge, 2015).

\section{Locomotion technique}

To travel in the VE, subjects used the KatWalk ODT. The KatWalk ODT is composed of a heavy base with a slippery curved platform (yellow in Figure 4), holding a rotating element with straps (blue on Figure 4) maintaining the user in the center of it, allowing full $360^{\circ}$ rotation on the $\mathrm{Z}$ axis. The main feature of this omnidirectional treadmill is its open construction that minimizes constraints on movement and allows users to 
perform all basic movements such as walking. While other omnidirectional treadmills put users inside a support ring, which stands in the way and prevents unrestricted movement of arms and legs, the KatWalk puts users inside a special harness. This harness can be adjusted to accommodate various body shapes with maximum carry capacity up to $308 \mathrm{lbs}(140 \mathrm{~kg})$. The support structure itself is designed to work with users measuring between 4'3" and to 6'7' $(130 \mathrm{~cm}-200 \mathrm{~cm})$. Once strapped in the harness, users can freely move without having to worry about hitting the supporting structure. Walking and running has a semi-realistic friction thanks to a pair of specially designed shoes ${ }^{1}$.

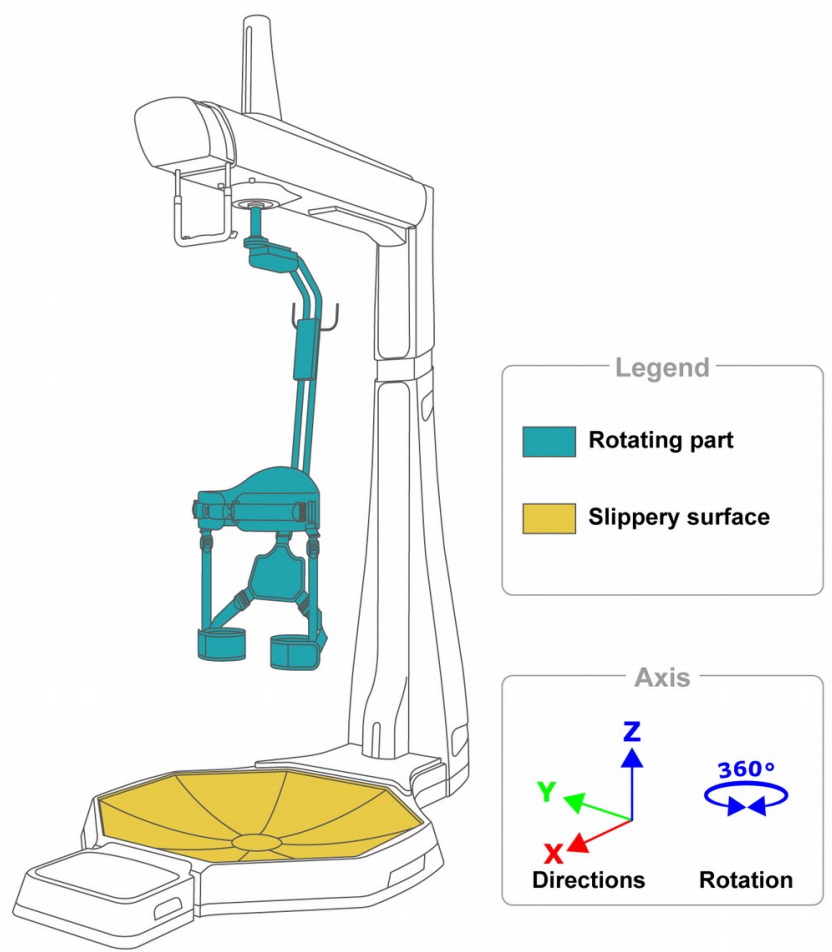

Figure 4. KatWalk omnidirectional treadmill geometry.

\section{Questionnaires}

Three questionnaires were used: 1/ an ad hoc questionnaire to measure the participants practice of VR tools, the participants level of technophilia and the ease of use about the VR tools after the task, 2/ SSQ test (Kennedy et al., 1993) and 3/ Self-Assessment Manikin (Bradley \& Lang, 1994). The ad hoc questionnaire was composed of three questions about the participants practice of VR tools, four questions about their level of technophilia (e.g., "In general, I do not hesitate to try new technologies") and four questions about the ease of use. For the technophilia and the ease of use, affirmations were presented and participants had to indicate on 6-point Likert scale (Joshi et al., 2015) their degree of agreement from 0 (= I don’t agree) to 5 (= I totally agree). The level of technophilia was then calculated thanks to the four questions scores.

The SSQ test (Kennedy et al., 1993) was composed of 16 symptoms (e.g., “fatigue”, "headache” or “eyestrain”) and participants had to indicate their level of feeling for each symptom on 4-point Likert scale from 0 (= I don't feel this symptom) to 3 (= I strongly feel this symptom). The objective of this questionnaire was to evaluate the evolution of the symptoms of motion sickness. The scores by symptoms were maintained and no aggregated measure was carried out.

1 Source: official site of the KatWalk: https://www.kat-vr.com/ 
The Self-Assessment Manikin (Bradley \& Lang, 1994; Estupiñán et al., 2014) evaluated 3 dimensions: the pleasure, the arousal and the dominance. For each dimension, 5 pictures are presented, and participants had to choose the picture that best represented their feelings. The responses were converted in 5-point Likert scale. The Self-Assessment Manikin is a tool created to observe emotional changes even over short exposures of the order of a few seconds. To our knowledge, VR studies have not used the SAM to assess emotional change but other tools, such as Allcoat and von Mühlenen, over a 7-minute exposure, have shown changes in the assessment of emotions during VR learning (Allcoat \& Muehlenen, 2018).

A free expression space was also placed at the end of the questionnaires.

\section{Procedure}

Before the task

Firstly, participants completed and signed a consent form to participate in this study. Then, a stereoscopic test (Randot test) was done to make sure they had a 3D vision. They filled out a questionnaire about: a/ their practice of VR tools, b/ their level of technophilia, c/ the SSQ test and d/ the Self-Assessment Manikin.

Familiarization task

Each session started with a five minutes familiarization phase. Subjects were asked to move freely in the virtual scene and to reach a highlighted point in a simple virtual environment.

The aim of this phase is to allow subjects to be rapidly able to use the KatWalk ODT to travel in the virtual world. Assessment task

After the familiarization task, the subjects accomplished an assessment task. Participants had to reach successively five highlighted points in the VE using the virtual reality treadmill to move (see ). They were instructed to walk as straight as possible, with no time pressure.

Two independent variables intra-subject were tested: the ground type (plan or bumpy) and the presence of obstacle (with or without); thus, four experimental conditions. Each subject accomplished the assessment task which is composed of 4 tests corresponding to 4 different conditions:

- Cond1: Walking on a ground with a plane surface without obstacle;

- Cond2: Walking on a ground with a bumpy surface without obstacle;

- Cond3: Walking on a ground with a plane surface in presence of obstacles;

- Cond4: Walking on a ground with a bumpy surface in presence of obstacles.

In order to eliminate any learning experience, the conditions change from one subject to another. The mean total time spent in VR (i.e., for the four conditions) was to 130.43 seconds ( $S D=75.05$ ). The total distance from the starting point to the final target is 105 meters, passing through all five targets for Cond 1 and Cond2, and 108 meters for Cond3 and Cond4. The closest distance between each point is considered as the optimal trajectory. The deviation of the participants' trajectories was measured according to optimal trajectory which consists in the succession of the different vectors linking the target points. The distance $d_{u}$ between each point of the user' trajectory and the optimal trajectory was calculated (in meter).

After the task

After the task, participants filled out again the SSQ test and the Self-Assessment Manikin. They also completed the four questions about the ease of use.

\section{Results}

All participants managed to find and reach every target, including targets 4 and 5, placed outside of direct field of view, forcing them to turn their body to see them (see Figur). No significant difference in the number of collisions 
was observed between the plan ground $(M=1.38, S D=2.67)$ and the bumpy ground $(M=1.79, S D=3.52)$, Mann-Whitney $U=1177.5, p=.825$. The ease of use and the appropriation of the KatWalk ODT are correctly evaluated by the participant $(M=3.61$ [on 5], $S D=1.36)$ and we observed a trend in the number of collisions between the first $(M=3.83, S D=4.32)$ and second steps $(M=2.50, S D=3.16)$ with obstacles (Wilcoxon $T$ test $=$ $50, p=.120)$. Moreover, no difference appeared for the collision between female $(M=2, S D=3.76)$ and male $(M$ $=1.09, S D=2.06)$, Mann-Whitney $U=1257, p=.326$.

By superimposing all trajectories of the participants (Figure), we can observe a relative proximity between the paths, independently of users' affinity or experience in VR. However, we observe a significant difference between our four conditions $(K(3, N=96)=9.395, p=.024)$, in particular between the condition "plane surface with obstacles" $(M=9.92, S D=6.37)$ and the condition "bumpy surface without obstacle" $(M=15.77, S D=7.64)$ in the movement deviation with the optimal trajectory $(p=.014)$. Moreover, the analyses confirmed a significant difference between the presence of obstacles $(M=11.27, S D=7.05)$ and their absence $(M=14.39, S D=7.27)$, Mann-Whitney $U=828.5, p=.018$, and a trend effect of the ground type on the movement deviation (plane surface: $M=11.46, S D=6.68$; bumpy surface: $M=14.2, S D=7.68 ; p=.053$ ).

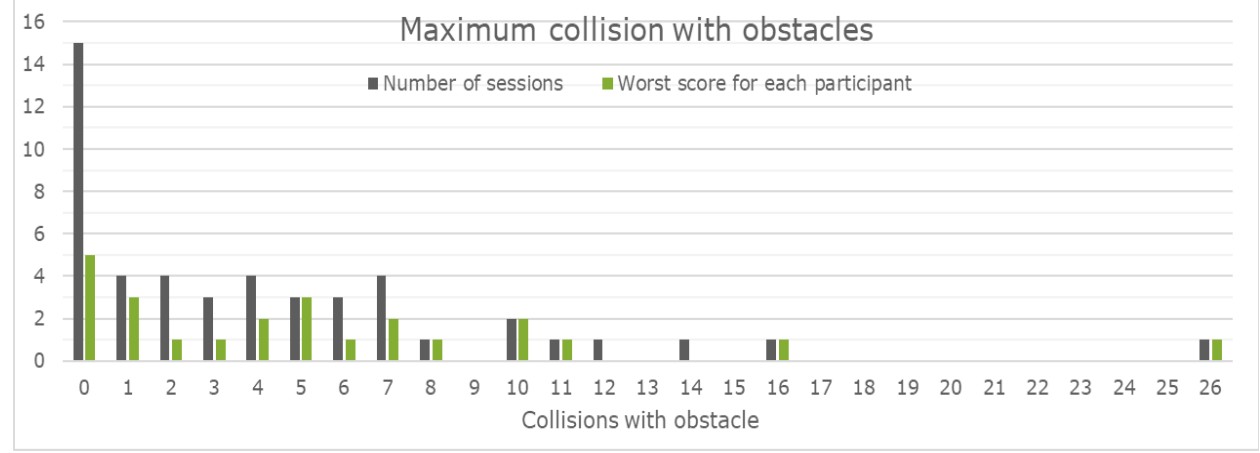

Figure 5. Maximum numbers of collisions per session and per participant.

For all 48 sessions with obstacles, 15 were performed without hitting any obstacle, and 33 with less than 5 collisions (see Figure 5). Five participants managed to touch no obstacle for any session, 15 made 5 or less collisions at most per session, and 21 participants made 10 or less. These collision scores are not correlated with technophilia ( $r=-.197, p=3.57$ ). Moreover, we observed no significative correlation between the technophilia and the affinity with the experiences on the ease of use or appropriation of the treadmill ( $p>.05$ for all correlations) even if a positive correlation trend appeared for the quick appropriation of the KatWalk ( $r=.369, p$ $=.076$ ). The level of technophilia and the speed of KatWalk appropriation varied in the same direction. Also, there was no correlation between the age or gender and other criteria ( $p>.05$ for all correlations).

Figure 6 shows a superposition of the participants' collisions with obstacles, highlighting a concentration at "tricky" passages for most of these, such as the curve between points 1 and 2, and the $270^{\circ}$ turns. 

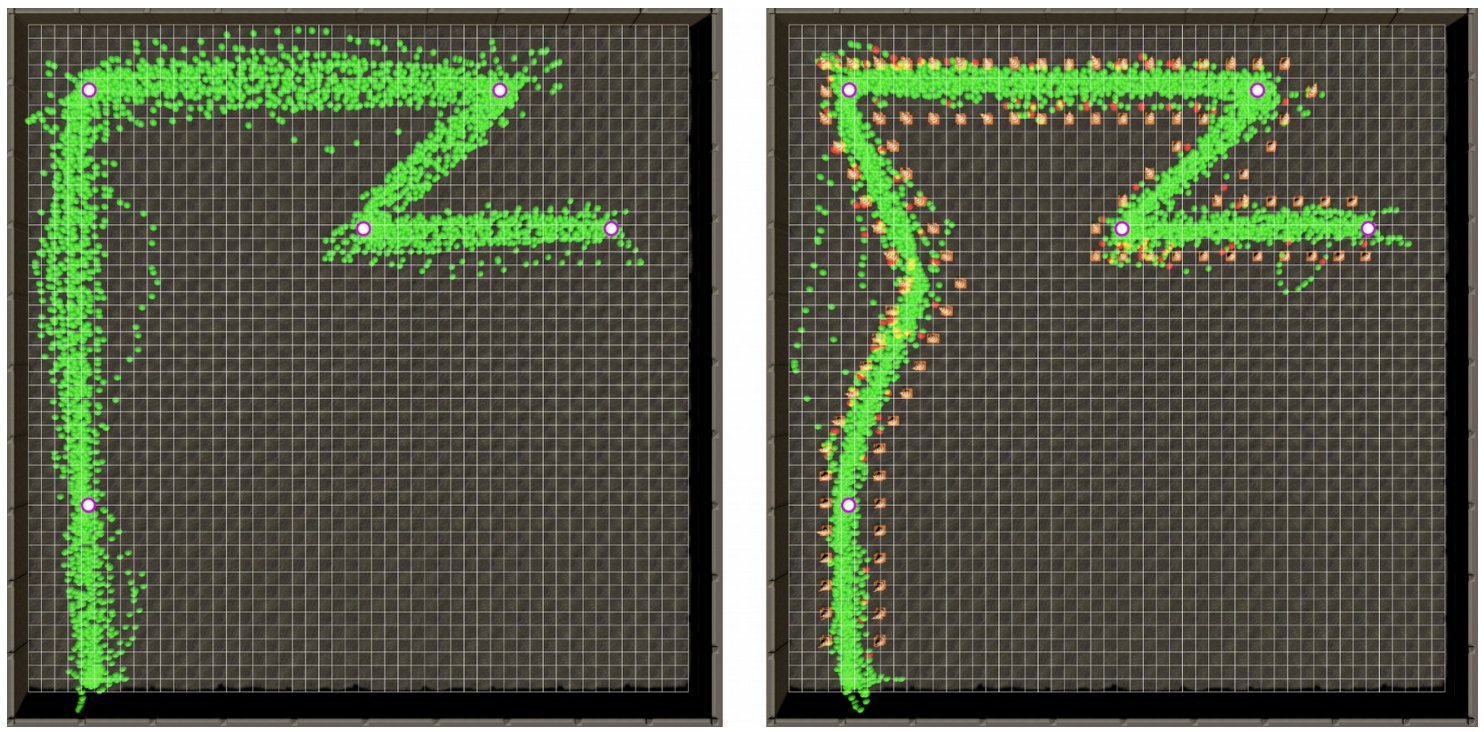

Figure 6. Superimposed plan and bumpy grounds trajectories of participants, without obstacle (left) and with obstacles (right). The grid's squares are one meter large.

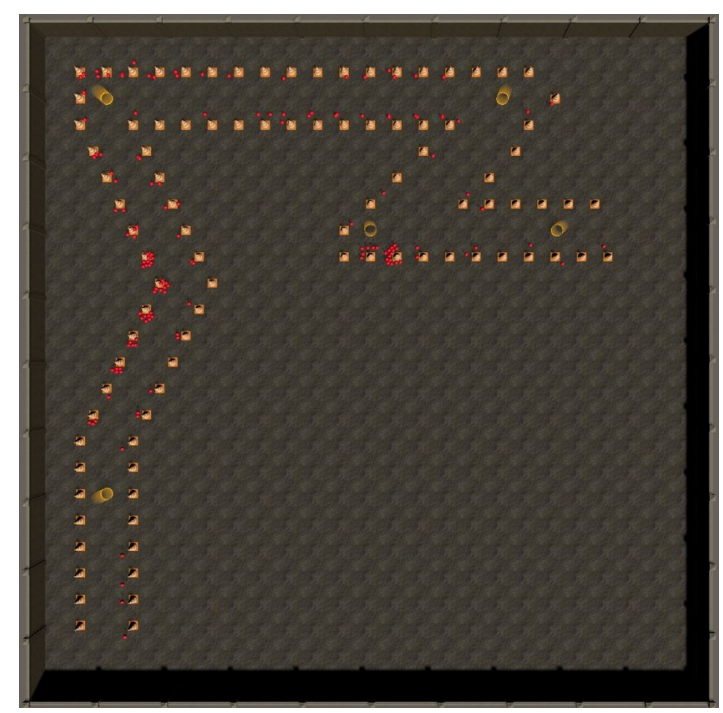

Figure 7. Location of collisions with obstacles for all participants.

Table 2. Means and Deviations for the SSQ test before and after the use of KatWalk ODT. 


\begin{tabular}{|c|c|c|c|c|c|c|c|c|}
\hline & \multicolumn{2}{|c|}{ GD* } & \multicolumn{2}{|c|}{$\mathbf{F}$} & \multicolumn{2}{|c|}{$\mathbf{H}$} & \multicolumn{2}{|c|}{$\mathbf{E}$} \\
\hline & $\mathrm{M}$ & $S D$ & $\mathrm{M}$ & $S D$ & $\mathrm{M}$ & $S D$ & $\mathrm{M}$ & $S D$ \\
\hline Before & .38 & .71 & 1.1 & .93 & .38 & .65 & .8 & .83 \\
\hline \multirow[t]{3}{*}{ After } & .96 & .81 & 1 & .81 & .58 & .65 & .6 & .65 \\
\hline & \multicolumn{2}{|c|}{ DF } & \multicolumn{2}{|c|}{ IS } & \multicolumn{2}{|c|}{$\mathrm{S}^{*}$} & \multicolumn{2}{|c|}{$\mathbf{N}^{*}$} \\
\hline & $\mathrm{M}$ & $S D$ & $\mathrm{M}$ & $S D$ & $\mathrm{M}$ & $S D$ & $\mathrm{M}$ & $S D$ \\
\hline Before & .29 & .69 & .08 & .28 & .29 & .55 & .29 & .69 \\
\hline \multirow[t]{3}{*}{ After } & .33 & .57 & .21 & .42 & .92 & .93 & .71 & .96 \\
\hline & \multicolumn{2}{|c|}{ DC } & \multicolumn{2}{|c|}{ FH* } & \multicolumn{2}{|c|}{ BV } & \multicolumn{2}{|c|}{ DEO } \\
\hline & $\mathrm{M}$ & $S D$ & M & $S D$ & $\mathrm{M}$ & $S D$ & $\mathrm{M}$ & $S D$ \\
\hline Before & .46 & .51 & .2 & .48 & .21 & .66 & .2 & .66 \\
\hline \multirow[t]{3}{*}{ After } & .42 & .65 & .8 & .79 & .38 & .65 & .2 & .51 \\
\hline & \multicolumn{2}{|c|}{ DEC } & \multicolumn{2}{|c|}{$\mathbf{V}^{*}$} & \multicolumn{2}{|c|}{ SA } & \multicolumn{2}{|c|}{ B } \\
\hline & $\mathrm{M}$ & $S D$ & $\mathrm{M}$ & $S D$ & M & $S D$ & $\mathrm{M}$ & $S D$ \\
\hline Before & .17 & .48 & .13 & .45 & .25 & .53 & .08 & .28 \\
\hline After & .13 & .34 & .54 & .93 & .58 & 1.02 & .38 & .92 \\
\hline
\end{tabular}

Notes. $G D=$ General Discomfort,$F=$ Fatigue, $H=$ Headache, $E=$ Eyestrain, $D F=$ Difficulty Focusing, IS = Increased Salivation, $S=$ Sweating, $N=$ Nausea, $D C=$ Difficulty Concentrating, $F H=$ Fullness of Head, $B V=$ Blurred Vision, $D E O=$ Dizzy (Eyes Open), DEC = Dizzy (Eyes Closed), $V=$ Vertigo, $S A=$ Stomach Awareness, $B$ = Burping. ${ }^{*} p<.05$

Concerning the SSQ test (see Table 2), we observed a significant increase between before and after the use of KatWalk ODT on general discomfort $(p=.008)$, sweating $(p=.016)$, nausea $(p=.019)$, vertigo $(p=.007)$ and fullness of head $(p=.004)$, and a trend on stomach awareness $(p=.071)$. There is no difference for the other symptoms $(p>.05)$.

On the other hand, no difference appeared for the Self-Assessment Manikin scores between before and after the use of KatWalk ODT ( $p>$.05). The participants had the same feelings between both evaluations (see Table 3).

Table 3. Means and Standard deviations for the three dimensions of Self-Assessment Manikin before and after the use of KatWalk ODT.

\begin{tabular}{lcccccc}
\hline & \multicolumn{2}{c}{ Pleasure } & \multicolumn{2}{c}{ Arousal } & \multicolumn{2}{c}{ Dominance } \\
\cline { 2 - 7 } & $\mathrm{M}$ & $S D$ & $\mathrm{M}$ & $S D$ & $\mathrm{M}$ & $S D$ \\
\hline Before & .83 & .82 & 2.29 & 1.27 & 2.33 & .96 \\
After & 1.04 & .91 & 2.29 & 1.2 & 2.38 & .97 \\
\hline
\end{tabular}

\section{Discussion}

The results of this study provide interesting reflections about the use of the KatWalk ODT in VR. Contrary to our hypotheses, the different conditions are not equivalent concerning the movement deviation from the optimal trajectory. Indeed, when there are obstacles, the participants deviate less from their trajectory. Obstacles appear to help participants to stay on their trajectory. A possible interpretation is that objects disposed in the environment help participants to have spatial references and consequently they are more aware of their own position and speed in this VE. Moreover, although this effect is only tendential, bumpy ground causes more deviation in the 
trajectory than when the ground is plane. A study with more participants should be able to confirm or not confirm this trend effect.

The decrease of the collisions, tending to be significant, between the two sessions with obstacles suppose that a break-in time was necessary for subjects to grip with the tool. The result was not significant but encourages to start with a familiarization session before experiments.

The proximity of the different paths supposes a good control of the locomotion technique, independently of previous experiences. The concentration of collisions areas also tends to show that they are more due to the environment difficulty than a loss of control, even if this study is not focus enough to produce a strong conclusion.

The lack of correlation between the technophilia and the affinity with the experiences on the ease of use or appropriation of the treadmill could be interpreted by the fact that there is no need for specific prior experience on new technologies to learn how to use the KatWalk ODT and manage to use it efficiently. Moreover, the lack of correlation between the gender or the age with the other variables could mean that the tool seems to be quite universal. However, it is important to highlight that the lack of correlation can also come from our sample size which is statistically small $(N=24)$.

In order to test the effects of the KatWalk ODT on feelings (i.e., Self-Assessment Manikin) and motion sickness (i.e., SSQ), we compared the scores of participants before and after they used the KatWalk ODT. If no significant result appears for SAM, the symptoms measuring the motion sickness are not the same between before and after a session. Indeed, for five symptoms (i.e., general discomfort, sweating, nausea, fullness of head and vertigo), the participants declared that they felt their symptoms more intensely after using the KatWalk ODT than before. The tool seems to cause motion sickness even after a short exposure (on average 130.43 seconds). It is important to note that, even though motion sickness is an on-going problem during a virtual reality experience with HMD, the symptoms of motion sickness we found are moderate and the scores did not exceed 1 on a scale ranging from 0 to 3 (Arcioni et al., 2019; Davis et al., 2014; Farmani \& Teather, 2018).

The carried study allowed us to validate our second hypothesis $\mathrm{H} 2$ and to confirm that the KatWalk ODT can be used as a LT for VR. Besides, it does not seem to interfere negatively on the immersion of participant and may have a positive influence on it. Nevertheless, the simplicity of the situations studied cannot confirm this hypothesis. The first hypothesis on the use of a bumpy ground in the VE is also confirmed: users were able to accomplish the task in the conditions cond2 and cond4 and did not report any particular problem with the obstacles. We also noticed that there was no significant difference between the number of collisions in the bumpy ground and the plane ground: participants walked in both conditions with equal ease.

In the next study, we plan to compare the KatWalk ODT with other common LT in VR (e.g., joystick). Related results will allow us to identify the advantages and disadvantages of each LT and then to list recommendations for the choice of the LT depending on the aim of the VR experiment. Further studies, including active tasks, could also show the possibilities and limitation of the ODT beyond exclusive locomotion.

\section{References}

Allcoat, D., \& Muehlenen, A. (2018). Learning in virtual reality: Effects on performance, emotion and engagement. Research in Learning Technology 26, (2018).

Arcioni, B., Palmisano, S., Apthorp, D., \& Kim, J. (2019). Postural stability predicts the likelihood of cybersickness in active HMD-based virtual reality. Displays 58, (July 2019), 3-11. doi:10.1016/j.displa.2018.07.001

Barberis, A., Bennet, T., \& Minear, M. (2019). "Ready Player One”: Enhancing Omnidirectional Treadmills for Use in Virtual Environments. In 2019 IEEE Conference on Virtual Reality and 3D User Interfaces (VR), 848-849.

Berger, L., \& Wolf, K. (2018). WIM: Fast Locomotion in Virtual Reality with Spatial Orientation Gain \& Without 
Motion Sickness. In Proceedings of the 17th International Conference on Mobile and Ubiquitous Multimedia (MUM’2018), 19-24.

Bowman, D.A., Kruijff, E., LaViola, J.J., \& Poupyrev, I. (2004). 3D User Interfaces: Theory and Practice. Addison Wesley Longman Publishing Co., Inc., USA.

Bozgeyikli, E., Raij, A., Katkoori, S., \& Dubey, R. (2016). Locomotion in Virtual Reality for Individuals with Autism Spectrum Disorder. In Proceedings of the 2016 Symposium on Spatial User Interaction (SUI '16), 33-42. doi:10.1145/2983310.2985763

Bozgeyikli, E., Raij, A., Katkoori, S., \& Dubey, R. (2016). Point \& Teleport Locomotion Technique for Virtual Reality. 205-216. doi:10.1145/2967934.2968105

Bozgeyikli, E., Raij, A., Katkoori, S., \& Dubey, R. (2019). Locomotion in virtual reality for room scale tracked areas. International Journal of Human-Computer Studies 122, (2019), 38-49. doi:10.1016/j.ijhcs.2018.08.002

Bradley, M.M., \& Lang, P.J. (1994). Measuring emotion: The self-assessment manikin and the semantic differential. Journal of Behavior Therapy and Experimental Psychiatry 25, 1 (1994), 49-59.

Bruder, G., Lubos, P., \& Steinicke, F. (2015). Cognitive Resource Demands of Redirected Walking. IEEE Transactions on Visualization and Computer Graphics 21, (2015), 539-544.

Bruder, G., Steinicke, F., Bolte, B., Wieland, P., Frenz, H., \& Lappe, M. (2013). Exploiting perceptual limitations and illusions to support walking through virtual environments in confined physical spaces. Displays 34, 2 (2013), 132-141.

Cherep, L., Lim, A., Kelly, J., Ostrander, A., \& Gilbert, S.B. (2020). Spatial cognitive implications of teleporting through virtual environments. doi:10.31234/OSF.IO/CX9VT

Cherni, H. (2012). Impact of virtual information characteristics in a task of search of targets : perspectives in cognitive rehabilitation. Ecole nationale supérieure d'arts et métiers - ENSAM. Retrieved from https://pastel.archives-ouvertes.fr/pastel-00866795

Cherni, H., Métayer, N., \& Souliman, N. (2020). Literature review of locomotion techniques in virtual reality. International Journal of Virtual Reality 20, 1 (2020), 1-20. doi:10.20870/IJVR.2020.20.1.3183

Davis, S., Nesbitt, K., \& Nalivaiko, E. (2014). A Systematic Review of Cybersickness. In Proceedings of the 2014 Conference on Interactive Entertainment - IE2014, 1-9.

Estupiñán, S., Rebelo, F., Noriega, P., Ferreira, C., \& Duarte, E. (2014). Can Virtual Reality Increase Emotional Responses (Arousal and Valence)? A Pilot Study. pp 541-549. doi:10.1007/978-3-319-07626-3_51

Farmani, Y., \& Teather, R.J. (2018). Viewpoint Snapping to Reduce Cybersickness in Virtual Reality. In Proceedings of the 44th Graphics Interface Conference (GI '18), 168-175. doi:10.20380/GI2018.23

Grechkin, T.Y., Plumert, J.M., \& Kearney, J.K (2014). Dynamic Affordances in Embodied Interactive Systems: The Role of Display and Mode of Locomotion. IEEE Transactions on Visualization and Computer Graphics 20, 4 (2014), 596-605. doi:10.1109/TVCG.2014.18

Jacob Habgood, M.P., Moore, D., Wilson, D., \& Alapont, S. (2018). Rapid, Continuous Movement Between Nodes as an Accessible VirtualReality Locomotion Technique. In 2018 IEEE Conference on Virtual Reality and 3D User Interfaces (VR), 371-378.

Hale, K.S., \& Stanney, K.M. (2014). Handbook of Virtual Environments: Design, Implementation, and Applications (2nd ed.). CRC Press, Inc., Boca Raton, FL, USA.

Jayaraman, S.K, Creech, C., Robert Jr., L.P., Tilbury, D., Yang, X.J., Pradhan, A.K., \& Tsui, K.M. (2012). Trust in AV: An Uncertainty Reduction Model of AV-Pedestrian Interactions, in Companion of the 2018 ACM/IEEE International Conference on Human-Robot Interaction, New York, NY, USA. 18, (2012), 626-633. 
Joshi, A., Kale, S., Chandel, S., \& Pal, D. (2015). Likert Scale: Explored and Explained. British Journal of Applied Science \& Technology 7, 4 (2015), 396-403.

Kennedy, R.S., Lane, N.E., Kevin, S., \& Lilienthal, M.G. (1993). The International Journal of Aviation Psychology Simulator Sickness Questionnaire: An Enhanced Method for Quantifying Simulator Sickness. The International Journal of Aviation Psychology 3, 3 (1993), 203-220.

Kitson, A., Hashemian, A.M., Stepanova, E.R., Kruijff, E., \& Riecke, B.E. (2017). Comparing leaning-based motion cueing interfaces for virtual reality locomotion. In 2017 IEEE Symposium on 3D User Interfaces (3DUI), 73-82. doi:10.1109/3DUI.2017.7893320

Kitson, A., Riecke, B.E., Hashemian, A.M., \& Neustaedter, C. (2015). NaviChair: Evaluating an Embodied Interface Using a Pointing Task to Navigate Virtual Reality. In Proceedings of the 3rd ACM Symposium on Spatial User Interaction (SUI '15), 123-126.

Lee, J., Ahn, S.C., \& Hwang, J.I., (2018). A Walking-in-Place Method for Virtual Reality Using Position and Orientation Tracking. Sensors (Basel, Switzerland) 18, 9 (September 2018).

Linn, A.M. (2017). Gaze Teleportation in Virtual Reality. KTH, School of Computer Science and Communication, Master Thesis.

McMahan, R., Bowman, D.A., Zielinski, D.J., \& Brady, R. (2012). Evaluating Display Fidelity and Interaction Fidelity in a Virtual Reality Game. IEEE transactions on visualization and computer graphics 18, (2012), 626633. doi:10.1109/TVCG.2012.43

Nabiyouni, M., Saktheeswaran, A., Bowman, D.A., \& Karanth, A. (2015). Comparing the performance of natural, semi-natural, and non-natural locomotion techniques in virtual reality. In 2015 IEEE Symposium on 3D User Interfaces (3DUI), 3-10. doi:10.1109/3DUI.2015.7131717

Nilsson, N.C., Serafin, S., Laursen, M.H., Pedersen, K.S., Sikström, E., \& Nordahl, R. (2013). Tapping-In-Place: Increasing the naturalness of immersive walking-in-place locomotion through novel gestural input. In 2013 IEEE Symposium on 3D User Interfaces (3DUI), 31-38. doi:10.1109/3DUI.2013.6550193

Ohshima, T., Ishihara, H., \& Shibata, R. (2016). Virtual ISU: Locomotion Interface for Immersive VR Gaming in Seated Position. In Proceedings of the 2016 Virtual Reality International Conference (VRIC '16), 2:1--2:4. doi:10.1145/2927929.2927941

Razzaque, S., (2005). Redirected Walking. University of North Carolina at Chapel Hill, Chapel Hill, NC, USA.

Slater, M., Usoh, M., \& Steed, A. (1995). Taking Steps: The Influence of a Walking Technique on Presence in Virtual Reality. ACM Trans. Comput.-Hum. Interact. 2, 3 (1995), 201-219.

Steinicke, F., Bruder, G., Jerald, J., Frenz, H., \& Lappe., M. (2010). Estimation of Detection Thresholds for Redirected Walking Techniques. IEEE Transactions on Visualization and Computer Graphics 16, 1 (2010), 1727. doi:10.1109/TVCG.2009.62

The verge (2015). Valve's VR headset is called the Vive and it's made by HTC.

Warren, L.E., \& Bowman, D.A. (2017). User Experience with Semi-natural Locomotion Techniques in Virtual Reality: The Case of the Virtuix Omni. In Proceedings of the 5th Symposium on Spatial User Interaction (SUI '17), 163.

Tunnell Wilson, P., Kalescky, W., MacLaughlin, A., \& and Williams, B. (2016). VR Locomotion: Walking > Walking in Place > Arm Swinging. In Proceedings of the 15th ACM SIGGRAPH Conference on Virtual-Reality Continuum and Its Applications in Industry - Volume 1 (VRCAI '16), 243-249.

Zank, M, \& Kunz, A. (2015). Using Locomotion Models for Estimating Walking Targets in Immersive Virtual Environments. In Proceedings of the 2015 International Conference on Cyberworlds (CW) (CW '15), 229-236. 
doi:10.1109/CW.2015.20 